\title{
Influência das Relações Familiares na Saúde e no Estado Emocional dos Adolescentes
}

\author{
Influence of Family Relations on the Health and Emotional State of Adolescents \\ Influencia de las Relaciones Familiares sobre la Salud y el Estado Emocional de los \\ Adolescentes
}

\author{
Patrícia Martins de Freitas ${ }^{1}$ \\ Universidade Federal da Bahia (UFBA) \\ Raphael Silva Nogueira Costa \\ Centro Universitário Dom Pedro /I \\ Marianna Santos Rodrigues \\ Universidade Federal da Bahia (UFBA) \\ Bruna Rafaela de Assis Ortiz \\ Júlio César dos Santos \\ Universidade Federal do Recôncavo da Bahia (UFRB)
}

\begin{abstract}
Resumo
A saúde dos adolescentes pode sofrer a influência de diferentes fatores, incluindo as relações familiares. Neste estudo, o objetivo foi verificar quais dimensões das relações familiares podem predizer o bem-estar durante a adolescência. Participaram 203 adolescentes, entre 12 e 18 anos, de escolas públicas de Santo Antônio de Jesus, Bahia. Os instrumentos utilizados foram: Inventário Beck de Depressão; Questionário de Saúde Geral de Goldberg; Inventário de Autoavaliação para Jovens; Familiograma; e Inventário do Clima Familiar. Para análise de dados, utilizaram-se estatística descritiva, comparação de grupos e análise de regressão múltipla. Os resultados demonstraram maior influência das dimensões conflito e baixa afetividade como variáveis que contribuem para a presença de sintomas depressivos, baixos escores de saúde geral e maiores escores de problemas de comportamento. Conclui-se que, para o bem-estar dos adolescentes, a família deverá ser incluída nas estratégias de avaliação e intervenção em saúde.

Palavras chave: relações familiares, saúde mental, adolescentes
\end{abstract}

\begin{abstract}
Adolescent health might be influenced by different factors, including family relationships. In this study, the objective was to verify which dimensions of family relationships might predict well-being during adolescence. The participants of the study were 203 adolescents, between 12 and 18 years, of public schools in Santo Antônio de Jesus, Bahia. The instruments used were: Beck Inventory of Depression; Goldberg General Health Questionnaire; Self-Assessment Inventory for Young People; Familiogram; and Family Climate Inventory. For data analysis, descriptive statistics, group comparison, and multiple regression analysis were used. The results showed a greater influence of conflict dimensions and low affectivity as variables that contribute to the presence of depressive symptoms, low general health scores, and higher scores of behavioral problems. It is concluded that, for the adolescents' well-being, the family should be included in health assessment and intervention strategies.
\end{abstract}

Keywords: family relationships, mental health, adolescents

\section{Resumen}

La salud del adolescente puede verse influenciada por diferentes factores, incluidas las relaciones familiares. En este estudio, el objetivo era verificar qué dimensiones de las relaciones familiares pueden predecir el bienestar durante la adolescencia. Participaron 203 adolescentes, entre 12 y 18 años, de escuelas de Santo Antônio de Jesus, Bahia. Los instrumentos: Inventario Beck de Depresión; Cuestionario de Salud General de Goldberg; Autoevaluación para Jóvenes; Familiograma; e Inventario del Clima Familiar. Para el análisis de datos, se utilizó estadística descriptiva, comparación de grupos y

\footnotetext{
1 Endereço de contato: Hormindo Barros, 58, Quadra 17, Lote 58, Bairro Candeias, CEP: 45029-094. E-mail: pmfrei@gmail.com
} 
regresión múltiple. Los resultados demostraron mayor influencia de las dimensiones conflicto y baja afectividad como variables que contribuyen a la presencia de síntomas depresivos, bajos escores de salud general y mayores escores de problemas de comportamiento. Se concluye que, para el bienestar de los adolescentes, la familia deberá ser incluida en las estrategias de evaluación y intervención en salud.

Palabras clave: relaciones familiares, salud mental, adolescentes

\section{Introdução}

A família é um sistema constituído de relações que formam uma rede participativa no desenvolvimento de crianças e adolescentes (McGoldrick, Garcia-Preto, \& Carter, 2016). As características da família e a natureza de suas relações podem funcionar como mecanismo de proteção, diminuindo a chance de problemas emocionais/comportamentais e de saúde. Por outro lado, algumas formas de relacionamento dentro da família, como o excesso de conflito e a baixa afetividade, podem funcionar como vulnerabilidade, aumentando o risco para saúde física e mental (Hess \& Falcke, 2013). O modelo teórico do desenvolvimento familiar direciona os mecanismos de desenvolvimento para as relações entre os membros da família ao longo do curso da vida, estabelecendo como indicadores as seguintes dimensões: Afetividade, que é definida como os vínculos afetivos entre os membros; Conflito, que representa as divergências entre os membros; e Hierarquia, que é a dimensão que caracteriza as relações de poder dentro do sistema familiar. A combinação entre essas dimensões produz alguns padrões, indicando se a família apresenta coesão, ou seja, se o sistema tem um perfil de relacionamento que preserva a individualidade dos membros e, ao mesmo tempo, forma uma unidade (McGoldrick et al., 2016). Muitas evidências já foram produzidas sobre a relação entre problemas emocionais e comportamentais, e alta frequência de conflitos na família durante a infância e a adolescência (Conceição \& Marques, 2012; De Matos et al., 2015; Pugh \& Farrell, 2012).

A perspectiva do desenvolvimento familiar contribui para evidenciar os parâmetros das relações interpessoais presentes na vida dos adolescentes que podem compor a vulnerabilidade emocional identificada nessa faixa etária. A vulnerabilidade emocional e os problemas de saúde mental aparecem como algumas das principais causas de mortalidade entre os adolescentes (World Health Organization [WHO], 2018), reforçando a importância de pesquisas que investiguem as causas desse fenômeno, que parece ter uma natureza multifatorial. Durante a adolescência, os jovens tendem a se afastar do sistema familiar com objetivo de fortalecer a formação da identidade. Esse afastamento ocorre simultaneamente a uma aproximação dos grupos de pares. A dinâmica das relações interpessoais na adolescência tem sido investigada com importante fator de explicação para os problemas de saúde emocional (Cardoso \& Malbergier, 2014). A organização do sistema familiar tem dinâmica própria, porém com maior regularidade de interação que acompanha o ciclo de vida dos membros e com alterações nas diferentes fases. A adolescência é uma etapa do desenvolvimento que tem como uma de suas tarefas a construção da identidade e a diferenciação das figuras parentais (McGoldrick et al., 2016; Melo \& Mota, 2013). Portanto, é esperado que o aumento dos conflitos e da dificuldade na comunicação ocorra nessa fase (Baumrind, 1978).

As relações envolvendo pais e filhos são reconhecidas como parte fundamental na construção da saúde emocional desses membros, tendo como função básica a proteção de seus filhos 
(McGoldrick et al., 2016; Melo \& Mota, 2013). Os laços afetivos favorecem o desenvolvimento emocional e previnem desajustes biopsicossociais, contribuindo na recuperação quando os desajustes são inevitáveis. Ao contrário, as famílias que têm como padrão de interação o excesso de conflitos e baixa afetividade criam um ambiente desencadeador de psicopatologias, como a depressão tanto para os filhos quanto para os pais (Cruvinel \& Boruchovitch, 2009).

Alguns estudos demonstram que relacionamentos seguros e estáveis com os pais são importantes para a saúde mental dos adolescentes (Conceição, 2012; Aggarwal \& Berk, 2015; Avanci, Assis, Oliveira, Ferreira, \& Pesce, 2007). Ainda sobre o perfil das relações familiares e a saúde mental de adolescentes, tem sido observado que não é a ausência de conflito que contribui para o bem-estar, sendo o conflito um aspecto da relação importante, especialmente quando conjugado com afetividade. Os conflitos são situações que permitem o desenvolvimento de estratégias de solução de problemas e aumento das habilidades sociais; portanto, também funcionalidade no desenvolvimento, os prejuízos para o bem-estar dos adolescentes são explicados pelo excesso dos conflitos (Silva \& Freire, 2014).

Outra consequência associada à dinâmica familiar tem sido o comportamento social desviante marcado por delinquência e agressividade, relacionado, geralmente, a um ambiente familiar com excesso de conflitos e baixa aceitação (Cardoso, Rodrigues, \& Vilar 2004; Dessen \& Polônia, 2007; Teodoro, Hess, Saraiva, \& Cardoso, 2014). No estudo de Malbergier, Cardoso e Amaral (2012), foram evidenciadas diferenças significativas entre as relações familiares dos adolescentes que consumiam álcool e dos que não consumiam, em que os adolescentes usuários de álcool apresentaram duas vezes mais chances de achar que os pais não se importavam com eles e de não ter um bom relacionamento com os pais, sendo identificada alta frequência de conflitos e baixa frequência de atividades entre os membros da família. Além dos comportamentos de risco, tem sido verificada maior ocorrência de problemas psiquiátricos, como depressão, transtornos alimentares e transtornos da ansiedade, em adolescentes que vivem em um ambiente familiar com baixa afetividade e muito controle (Baptista, Baptista, \& Dias, 2001; Galambos, Leadbeater, \& Barker, 2004).

De acordo com estudos realizados nos Estados Unidos da América (EUA), em Portugal e no Brasil, houve um aumento dos casos de adolescentes com depressão, assim como nas estatísticas mundiais (Rosa, Oureiro, \& Sequeira, 2018; Reppold \& Hutz, 2003; Cardoso et al., 2004; WHO, 2018). E os resultados de tais estudos sugerem que a investigação da depressão nessa fase deve ser mais explorada, investigando as possíveis causas e variáveis relacionadas com este fenômeno. A depressão em adolescentes é um problema de saúde pública, por ser um transtorno de alta prevalência e preditor de suicídio; por isso, deve ser amplamente investigado (Martin, Hernandez-Martinez, Acosta, \& Domenech-Llaberia, 2011).

No estudo de revisão de Hess e Falcke (2013), foram identificados apenas dois estudos no Brasil, investigando a sintomatologia internalizante e as relações familiares em adolescentes, demonstrando uma real escassez de pesquisas que façam a análise da dimensão emocional. A sintomatologia internalizante, caracterizada por respostas emocionais que não são expressas para o ambiente externo, estão correlacionadas, muitas vezes, com sintomas convergentes com os transtornos da ansiedade e da depressão.

Para reduzir essa limitação e ampliar o conhecimento científico sobre a influência das relações familiares para o estado de saúde física e emocional dos adolescentes, foi realizado o presente estudo. O objetivo foi verificar a influência de variáveis das relações familiares 
como afetividade, coesão e hierarquia para o bem-estar físico e psicológico dos adolescentes, tendo como medidas de variáveis: estresse, depressão e comportamento social. A hipótese testada foi: as relações familiares influenciam a saúde física e emocional dos adolescentes.

\section{Método}

\section{Participantes}

Os participantes foram convidados a partir de reuniões realizadas nas escolas com os adolescentes e suas famílias. As reuniões foram feitas em seis escolas públicas da região urbana da cidade de Santo Antônio de Jesus, estado da Bahia (BA). Apesar de ter sido feito o sorteio das escolas participantes, não houve nenhum critério de aleatorização dos adolescentes, portanto a amostra foi constituída pelos participantes que desejaram colaborar com o estudo. A amostra foi composta de 203 adolescentes, com idades entre 12 e 18 anos, sendo todos de escolas públicas da cidade. Os participantes pertenciam a famílias com até três salários mínimos de renda per capita. A média de idade foi de 16,03 anos ( $D P=2,03$ ). A maioria dos participantes era do sexo feminino $(73,4 \%)$. A distribuição da escolaridade mostra maior concentração para o ensino médio incompleto $(43,4 \%)$ e ensino fundamental incompleto $(46,5 \%)$.

\section{Instrumentos}

Familiograma: O instrumento avalia a percepção da afetividade e o conflito familiar nas díades familiares. Por meio de uma folha na qual são listados adjetivos, o participante escolhia a resposta devida utilizando uma escala Likert de cinco pontos. A escolha varia de "De jeito nenhum" a "Completamente", sendo que tal adjetivo corresponde à relação de uma díade específica, por exemplo: "Meu pai e minha mãe têm um relacionamento". A partir dos resultados, pode-se classificar as famílias em diferentes categorias: tipo I, famílias que apresentam alta afetividade e baixo conflito; tipo II, famílias com alta afetividade e alto conflito; tipo III, baixa afetividade e baixo conflito; e tipo IV, baixa afetividade e alto conflito. No presente estudo, foram consideradas as dimensões da afetividade e conflito para as díades entre adolescente-mãe, adolescente-pai e adolescente-irmã (o). O Familiograma tem uma estrutura bifatorial, com índice de confiabilidade, por meio do alfa de Cronbach, de 0,87 a 0,97 para amostra de universitários e de 0,87 a 0,93 para amostra de adolescentes. 0 instrumento apresenta validação por intermédio de análise fatorial confirmatória (Teodoro, 2006).

Inventário de Clima Familiar (ICF): O ICF tem 22 itens para avaliar a percepção do clima familiar, as dinâmicas existentes nos relacionamentos intra e extrafamiliar, considerando quatro fatores: apoio, coesão, conflito e hierarquia. O Fator Apoio apresenta itens que descrevem o suporte emocional e material entre membros da família; o Fator Coesão avalia o vínculo emocional existente entre os membros da família; já o Fator Conflito avalia a relação agressiva e conflituosa existente entre os membros da família; e o último fator, Hierarquia, representa o nível de controle e poder no sistema familiar (Teodoro, Allgayer, \& Land, 2009). O nível de fidedignidade do ICF para a percepção das mães variou entre 0,60 e 0,89. A validade foi verificada por meio da análise fatorial exploratória e confirmatória, com distribuição dos fatores e suas cargas fatoriais satisfatórias, além de evidências de correlações entre os fatores (Teodoro et al., 2009). 
Inventário de Autoavaliação para Jovens - Youth Self-Report - 11 a 18 anos (YSR): O YSR fornece uma apreciação global sobre o comportamento na perspectiva dos próprios adolescentes. O inventário pode ser dividido em duas partes: Competências Sociais e Problemas de Comportamento. Para o presente estudo, foi utilizada apenas a parte de problemas de comportamento, considerando o escore total, que é calculado a partir da soma dos 105 itens. A pontuação é obtida pela soma das respostas escolhidas pelo adolescente para cada item, utilizando uma escala Likert de três pontos ("não é verdadeira", "algumas vezes verdadeira" ou "muito verdadeira/frequentemente verdadeira"). Quanto maior o escore, mais problemas de comportamento foram relatados (Achenbach \& Rescorla, 2001; Rocha, Araújo, \& Silvares, 2008). O YSR ainda está em fase de validação para o Brasil e os resultados preliminares demonstram níveis de confiabilidade adequados, variando de 0,57 a 0,85 (alfa de Cronbach). Para este estudo, foram utilizadas as normas americanas, considerando que as normas brasileiras ainda não estão disponíveis.

Inventário de Depressão de Beck (BDI): Trata-se de um instrumento de autorrelato constituído por 21 grupos de afirmações. Cada grupo tem quatro afirmações em que a pessoa deve escolher a alternativa que descreva melhor a maneira como tem se sentido na última semana, incluindo o dia da aplicação. Estas afirmações estão relacionadas com sinalizações dos sintomas referentes a estados depressivos da pessoa. O escore é dado pela soma dos escores de cada item, variando de 0 a 3, sendo o escore máximo da escala de 63. Segundo Cunha (Cunha, 2001), o BDI é uma medida de intensidade da depressão, utilizada para rastreio de sintomas depressivos na população psiquiátrica e população geral. Na versão brasileira, foram desenvolvidos estudos para sujeitos a partir de 12 anos. O estudo psicométrico do instrumento está descrito no manual, sendo a fidedignidade analisada por meio do alfa de Cronbach, que variou de 0,70 a 0,82 para diferentes amostras não clínicas e clínicas; na técnica de teste-reteste, a correlação foi de 0,95. A validade foi evidenciada por validade de conteúdo e também foi verificada a convergência com outras escalas de depressão. As normas brasileiras utilizam um ponto de corte de acima de 18 pontos como indicativo de sinais clínicos de depressão para grupos não clínicos.

Questionário de Saúde Geral de Goldberg (QSG): A avaliação subjetiva do estado de saúde foi realizada com aplicação do QSG, que fornece uma perspectiva sobre o seu estado de saúde mental. O QSG consiste em 60 itens, aferidos conforme uma escala com quatro graus, que varia de: 1) "não absolutamente", 2) "não mais do que de costume", 3) "um pouco mais do que de costume", até 4) "muito mais do que de costume". Além do escore geral, os resultados do QSG originam cinco escores parciais gerados a partir dos fatores que constituem o instrumento. Os fatores e seus respectivos pontos de corte para o sexo feminino e masculino são: Fator I - Estresse ( II - Desejo de Morte (sexo feminino = 2,00; masculino =2,14); Fator III - Desconfiança no Próprio Desempenho (sexo feminino = 2,61; masculino =2,47); Fator IV - Distúrbios do Sono ( sexo feminino $=2,67$; masculino $=2,33$ ); Fator $V$ - Distúrbios Psicossomáticos (sexo feminino = 2,60; masculino = 2,20); e Saúde Geral (sexo feminino =2,42; masculino =2,33). O QSG foi validado para o Brasil, demonstrando confiabilidade variando entre 0,80 e 0,95 para os instrumentos. A validação fatorial foi realizada, assim como a validade de critério (Pasquali, Gouveia, Andriola, Miranda, \& Ramos, 1996). Atualmente, a validação do QSG precisa de novos estudos para atualização das normas. 


\section{Procedimentos}

A pesquisa foi aprovada pelo Comitê de Ética da Liga Baiana Contra o Câncer (LBCC), do Hospital Aristides Maltez, sob registro de número 209/08, atendendo às exigências da Resolução 466/2012 e 510/2016, do Conselho Nacional de Saúde (CNS). Os participantes foram convidados após uma reunião explicativa sobre o projeto, recebendo o Termo de Consentimento Livre e Esclarecido (TCLE), que foi assinado pelo adolescente e pelos pais. A aplicação foi realizada de forma coletiva no ambiente da escola, sempre com a presença de quatro aplicadores para esclarecer as possíveis dúvidas dos adolescentes. A aplicação coletiva foi adotada para facilitar a coleta com um número maior de participantes e, ao mesmo tempo, permitir que os adolescentes respondessem com maior veracidade a partir de uma avaliação com menor exposição, reduzindo, assim, o efeito de inibição diante de um aplicador. Antes do início da aplicação, os adolescentes eram orientados sobre como deveriam preencher os instrumentos e sobre a importância de tais informações.

\section{Análise de Dados}

A análise de dados foi realizada utilizando o programa estatístico Statistical Packge of Social Science (SPSS) versão 18. A primeira etapa foi a análise da normalidade da amostra, aplicando o teste de Kolmogorov-Smirnov, com resultados compatíveis com a distribuição normal $p>0,05$. As ferramentas utilizadas foram: estatística descritiva, comparação de grupos e regressão múltipla com o método stepwise (Mingotti, 2005).

\section{Resultados}

A análise descritiva demonstrou que os resultados médios não estão acima dos pontos de corte dos instrumentos utilizados. Os resultados sobre o perfil familiar e emocional dos adolescentes são apresentados na Tabela 1. Os valores gerais não são indicativos de problemas familiares ou problemas emocionais, pois as médias apresentadas pela amostra ficaram abaixo do ponto de corte dos instrumentos utilizados.

\section{Tabela 1}

Estatística descritiva dos escores dos testes utilizados para medir as relações familiares, a saúde física e emocional dos adolescentes

\begin{tabular}{|c|c|c|c|c|c|}
\hline Instrumentos & Fatores & Mínimo & Máximo & Média & DP \\
\hline \multirow[t]{4}{*}{ Inventário do Clima Familiar } & Conflito Familiar & 4 & 27 & 10,82 & 5,48 \\
\hline & Hierarquia & 7 & 30 & 16,67 & 4,68 \\
\hline & Apoio & 5 & 25 & 16,27 & 4,81 \\
\hline & Coesão & 5 & 25 & 17,25 & 5,27 \\
\hline \multicolumn{6}{|l|}{ Familiograma } \\
\hline & Afetividade (eu e mãe) & 1,00 & 55,00 & 41,05 & 10,55 \\
\hline & Conflito (eu e mãe) & 9,00 & 45,00 & 18,40 & 6,96 \\
\hline & Afetividade (eu e pai) & 1,00 & 55,00 & 35,60 & 12,12 \\
\hline & Conflito (eu e pai) & 9,00 & 44,00 & 20,30 & 9,09 \\
\hline & Afetividade (eu e irmã[o]) & 1,00 & 55,00 & 35,12 & 11,83 \\
\hline & Conflito (eu e irmã[o]) & 9,00 & 50,00 & 21,38 & 9,44 \\
\hline
\end{tabular}




\begin{tabular}{lccccc}
\hline \multicolumn{1}{c}{ Instrumentos } & Fatores & Mínimo & Máximo & Média & DP \\
\hline & Conflito (eu e família) & 7,00 & 41,00 & 19,50 & 8,43 \\
Inventário Beck de Depressão & & 0 & 37 & 9,31 & 8,66 \\
Questionário de Saúde Geral & Estresse & 1,00 & 3,56 & 1,64 &, 58 \\
& Desejo de Morte & 1,00 & 3,50 & 1,33 &, 53 \\
& Preocupação Desempenho & 1,00 & 3,41 & 1,66 &, 47 \\
& Perturbações do Sono & 1,00 & 4,00 & 1,50 &, 63 \\
& Somático & 1,00 & 3,00 & 1,60 &, 49 \\
& Geral de Saúde & 1,00 & 3,04 & 1,61 &, 45 \\
\hline
\end{tabular}

Legenda: Mínimo - escore mínimo no instrumento e nos fatores; Máximo - escore máximo obtido no instrumento e nos fatores; DP - Desvio Padrão.

Após verificar o perfil geral dos participantes, foi realizada a identificação do percentual de adolescente com resultados superiores aos pontos de corte e, portanto, com indícios de alterações no estado emocional. Os adolescentes investigados apresentaram um percentual de indivíduos compatíveis com perfil clínico, variando de $0 \%$ a 20,6\%, considerando os diferentes instrumentos utilizados. Esse percentual não é irrelevante, por se tratar de indivíduos da população não clínica, e está compatível com os dados da Organização Mundial de Saúde, os quais mostram que $10 \%$ a $20 \%$ dos adolescentes apresentam depressão (WHO, 2018). Tais resultados podem ser visualizados na Tabela 2, que compara o percentual entre o gênero masculino e feminino.

Tabela 2

Frequência de adolescentes acima dos pontos de corte para os fatores do Questionário de Saúde Geral e para o Inventário Beck de Depressão, comparando gênero masculino e feminino

\begin{tabular}{lcc}
\hline & Feminino (\%) & Masculino (\%) \\
\hline Estresse & $2(3,4)$ & $3(8,8)$ \\
Desejo de Morte & $8(13,8)$ & $3(8,8)$ \\
Preocupação com o Desempenho & $0(0)$ & $4(11,8)$ \\
Perturbações do Sono & $3(5,3)$ & $4(11,8)$ \\
Somático & $3(5,2)$ & $7(20,6)$ \\
Geral de Saúde & $1(1,7)$ & $4(11,8)$ \\
BDI & $1(2,3)$ & $5(8,2)$ \\
\hline
\end{tabular}

Legenda: Fatores do Questionário de Saúde Geral - Fator 1: Estresse; Fator 2: Desejo de Morte; Fator 3: Preocupação com o Desempenho; Fator 4: Distúrbios do sono; Fator 5; Distúrbios Somáticos; e Fator 6: Saúde Geral; BD: Inventário Beck de Depressão.

Os resultados descritivos sobre o percentual de adolescentes acima do ponto de corte demonstram que o sexo masculino apresenta maior percentual para todas as medidas, com exceção do Fator II Desejo de Morte, no qual as adolescentes apresentaram maior percentual. Os resultados médios do QSG e BDI foram comparados por meio do teste t de Student para testar diferenças entre o sexo, e não foram encontradas diferenças significativas. No $B D I$, o resultado para o sexo feminino foi $M=8,33$; $D P=6,96$; e para o masculino, $M=11,14$; $\mathrm{DP}=11,07$ [t $(198)=-1,36 \mathrm{p}=0,17]$, sendo o ponto de corte 18 pontos. Para o QSG, foram encontrados os seguintes valores médios: sexo feminino, $M=1,58$; $D P=0,40$; e masculino, 
$M=1,63 ; D P=0,48$ [t (197) $=-0,48 ; p=0,62]$, sendo o ponto de corte 2,42 para o sexo feminino e 2,33 pontos para o masculino.

Outra análise realizada foi a comparação entre a percepção dos adolescentes sobre as relações familiares, verificando possíveis diferenças entre o sexo. A comparação entre o sexo masculino e feminino foi feita por meio do teste t de Student. Considerando as dimensões das relações familiares, os resultados foram os seguintes: Coesão - sexo feminino, $M=17,35$; $D P=5,31$, e masculino, $M=16,69 ; D P=5,21[t=0,55 ; p=0,58]$; Conflito - para o feminino, $M=11,69 ; D P=6,04$, e masculino, $M=9,91 ; D P=4,83[t=1,45 ; p=0,14]$; Hierarquia - para o sexo feminino, $M=16,98 ; D P=5,11$, e masculino, $M=15,78 ; D P=3,94[t=1,17 ; p=0,24]$; e Clima Familiar - para o sexo feminino, $M=61,90$; $D P=14,30$, e para o masculino, $M=$ 58,$38 ; \mathrm{DP}=10,55[\mathrm{t}=1,26 ; \mathrm{p}=0,21]$. Não foram encontradas diferenças significativas para nenhuma das dimensões comparadas.

A análise de regressão múltipla foi realizada a partir do método stepwise para testar quais as variáveis contribuem ao estado emocional e à saúde geral dos adolescentes. Tanto para a saúde emocional quanto geral, todas as variáveis que caracterizam as relações familiares foram incluídas (ICF, Familiograma). Os resultados encontrados na análise de regressão múltipla demonstram que as variáveis relativas às relações familiares podem explicar o estado emocional e a saúde geral dos adolescentes participantes da pesquisa. Foram realizadas três análises: a primeira para verificar as variáveis que contribuem para a saúde geral dos adolescentes. O modelo com melhor ajuste apresentou uma variância explicada de $28 \%$, com contribuições das seguintes dimensões das relações familiares: Fator Conflito do Inventário do Clima Familiar e o Fator Hierarquia do Inventário do Clima Familiar $\left[R=0,53\right.$, adj $R^{2}=0,28$; $(p=0,02)]$. Os valores de Beta $(\beta)$ mostram que, quanto maior o conflito, maiores os escores no QSG $(\beta=0,47)$; ou seja, quanto maior o conflito na família, pior o estado de saúde dos adolescentes. Já a relação com a Hierarquia é invertida, sendo que, quanto maior a hierarquia, menor os escores no QSG e, portanto, melhores índices de saúde geral $(\beta=-0,31)$ (vide Tabela 3).

Tabela 3

Regressão múltipla das variáveis das relações familiares como preditoras da saúde geral dos adolescentes

\begin{tabular}{ccccccc}
\hline Variável Critério & Modelos & Variáveis & $\mathbf{R}$ & $\mathbf{R}^{\mathbf{2}}$ & $\mathbf{T}$ & $\mathbf{p}$ \\
\hline & 1 & Fator Conflito & 0,40 & 0,16 & 2,54 & 0,01 \\
Saúde Geral QSG & 2 & Fator Conflito & & & 3,42 & 0,00 \\
& & Fator Hierarquia & 0,53 & 0,28 & $-2,28$ & 0,02 \\
\hline
\end{tabular}

Legenda: Saúde Geral QSG (variável dependente) - Modelo 1: Fator Conflito; Modelo 2: Conflito e Hierarquia, explica $28 \%$ da variância.

Os sintomas depressivos foram mais bem explicados pelas variáveis Fator Apoio e Afetividade entre a díade "eu e minha mãe", com uma variância explicada de $52 \%$ para esse modelo $\left[R=0,72\right.$, adj $\left.R^{2}=0,52 ;(p=0,03)\right]$ (vide Tabela 4). O valor de Beta ( $\beta$ ) mostra que, quanto maiores as variáveis Apoio $(\beta=-0,42)$ e Afetividade $(\beta=-0,33)$, menos sintomas depressivos. Essa relação tem um poder explicativo satisfatório e com significância estatística. Para os problemas de comportamento verificados por meio da regressão, a variável que 
melhor explicou o modelo foi o Fator Conflito do Inventário do Clima Familiar, sendo o valor $51 \%$ de variância explicada $\left[R=0,71\right.$, adj $\left.R^{2}=0,51 ; \beta=0,44(p=0,02)\right]$ (vide Tabela 4). Isso significa que, quanto maior o conflito, maiores os problemas de comportamento. As outras dimensões das relações familiares não tiveram contribuições significativas para permanecer como parte da explicação dos problemas de comportamento.

Tabela 4

Regressão múltipla dos sintomas depressivos e dos problemas de comportamento, adotando as variáveis das relações familiares como preditoras

\begin{tabular}{|c|c|c|c|c|c|c|}
\hline Variável Critério & Modelos & Variáveis & $\mathbf{R}$ & $\mathbf{R}^{2}$ & $\mathbf{t}$ & $p$ \\
\hline \multicolumn{7}{|l|}{ Sintomas Depressivos BDI } \\
\hline & 1 & Fator Apoio & 0,58 & 0,34 & $-2,99$ & 0,01 \\
\hline & 2 & Fator Apoio & & & $-4,11$ & 0,00 \\
\hline & & Afetividade Eu e Mãe & 0,72 & 0,52 & $-2,46$ & 0,03 \\
\hline Problemas de comportamento YSR & 1 & Fator Conflito & 0,71 & 0,51 & 3,07 & 0,02 \\
\hline
\end{tabular}

\section{Discussão e Conclusões}

O objetivo do estudo foi investigar o perfil emocional e a saúde geral dos adolescentes, considerando a influência das relações familiares. Para isso, foram definidas como variáveis que podem influenciar o bem-estar físico e psicológico as seguintes dimensões: Afetividade, Hierarquia, Conflito e Clima Familiar. Os resultados encontrados evidenciam dois eixos de análise, o primeiro é perfil emocional e de saúde dos adolescentes que participaram do estudo, evidenciando um importante problema de saúde pública a partir de um percentual convergente com as estatísticas mundiais sobre os problemas de saúde mental na adolescência, e o segundo é o poder preditivo das variáveis familiares sobre a saúde física e mental nesta fase do desenvolvimento.

Em relação ao perfil emocional, os resultados da análise exploratória demonstram que o perfil emocional dos participantes não é indicativo de psicopatologias com resultados médios para os adolescentes e suas relações familiares fora dos pontos de corte para padrão clínico. Esse resultado já era esperado, pois, ao convidarmos os adolescentes nas escolas, estamos diante de uma amostra não clínica. Entretanto, ao investigarmos o percentual de participantes acima do ponto de corte, o resultado foi expressivo; por exemplo, para o Fator Desejo de Morte, com 13,8\% das meninas acima do ponto de corte, e para o Fator Somático, $20,6 \%$ dos meninos acima do ponto de corte. A distribuição de jovens acima do ponto de corte foi de $8,2 \%$ para meninas e 2,3\% para meninos. Para essa análise, foi realizada a comparação entre os sexos de forma exploratória, demonstrando piores resultados para o sexo masculino nas dimensões do QSG, em que foi registrado maior percentual de indivíduos acima do ponto de corte, com exceção do fator Desejo de Morte, no qual o sexo feminino apresentou um percentual maior do que o sexo masculino. 
Em relação ao BDI, o resultado percentual masculino é maior que o percentual feminino. Esses resultados demonstram que os sintomas depressivos foram identificados como presentes para ambos os sexos, porém em diferentes instrumentos. Esse resultado também foi encontrado no estudo de Justo e Calil (2006), demonstrando que apenas a diferença de gênero não é suficiente para explicar a presença de sintomas de depressão. Para os adolescentes, as especificidades da fase de desenvolvimento têm impactos mais fortes do que o gênero. No presente estudo, o resultado sobre características depressivas no sexo masculino e feminino foi encontrado por diferentes instrumentos, o que pode ser explicado pela forma menos direta de investigação do BDI, considerando que ele é um instrumento de triagem e que, portanto, permite a identificação de sintomas da depressão. Desta forma, os adolescentes do sexo masculino podem ter se sentido mais à vontade para responder ao BDI do que às questões do QSG que abordam o desejo de morte. Além disso, o Fator Desejo de Morte do QSG representa um indicador de que o sexo feminino apresentou mais vulnerabilidade para o suicídio. O perfil emocional e a percepção sobre a própria saúde dos adolescentes podem constituir um conjunto de variáveis que contribuem para os problemas como a socialização, autorregulação das emoções e desempenho escolar.

O perfil encontrado para o sexo masculino demonstra uma vulnerabilidade emocional acima do esperado, considerando que as estatísticas mundiais sobre o estado depressivo em homens são de 5,8\%, e nas mulheres, de 9,2\% (WHO, 2018). Esse resultado indica a importância de intervenções que atuem diretamente considerando demandas emocionais. As intervenções devem considerar não apenas o adolescente, mas também outros nichos sociais que interferem em seu bem-estar. Como tem sido demonstrado, os vínculos familiares fortes funcionam como fator de proteção para reduzir as chances do envolvimento de jovens com as drogas e criminalidade, sendo estas alterações comportamentais mais frequentes no sexo masculino (Hoffmann \& Cerbone, 2002; Schenker \& Minayo, 2003).

As dificuldades emocionais durante adolescência podem provocar prejuízos importantes para a vida adulta; por exemplo, adolescentes que são diagnosticadas como depressivas têm maior probabilidade de apresentarem comorbidades (outros transtornos associados) e serem hospitalizadas em enfermarias psiquiátricas quando adultas (Crandall, Ghazarian, Day, \& Riley, 2016; Baptista et al., 2001; Loureiro, Sequeira, Rosa, \& Gomes, 2014). No Brasil, estudos investigando a sintomatologia da depressão em adolescentes demonstram percentuais expressivos variando de 5\% a 22\% (Baptista et al., 2001; Ferreira, 2011). Os dados epidemiológicos demonstram que o aspecto emocional dos adolescentes é um importante foco de investigação, entretanto, é importante considerar as dimensões das relações familiares associadas a esse fenômeno.

Outro eixo de análise, sobre a influência das relações familiares sobre variáveis emocionais e de saúde física, indicou que a afetividade é uma dimensão bastante preditiva do perfil emocional. Os distúrbios psicossociais têm forte relação com problemas familiares que comprometem o desenvolvimento individual. Diversos estudos evidenciaram problemas emocionais e comportamentais em adolescentes que tinham famílias conflituosas (Henneberger, Varga, Moudy, \& Tolan, 2016; Rozemberg, Avanci, Schenker, \& Pires, 2014; Teodoro et al., 2014). A qualidade da relação entre pais e filhos pode favorecer as adaptações necessárias para a fase da adolescência. Por um lado, a qualidade de vida dos adolescentes pode estar relacionada ao bom relacionamento com os pais; por outro lado, suas habilidades sociais 
podem ser prejudicadas a partir de ambiente no qual o relacionamento familiar é predominantemente conflituoso (Conceição \& Marques, 2012). Além disso, quando a família não oferece um ambiente propício para o desenvolvimento emocional, as chances de este adolescente apresentar problemas de ordem psiquiátrica são maiores, com persistência dos problemas para a vida adulta (Baptista et al., 2001; Cardoso et al., 2004). Existem correlações entre o funcionamento familiar e o comportamento individual, sendo uma das consequências já evidenciadas a relação entre indivíduos com comportamentos disfuncionais e famílias com níveis altos de estresse psicológico (De Matos et al., 2015; Martin et al., 2011).

A relação entre o desenvolvimento psicossocial dos adolescentes e as interações familiares têm sido evidenciada em outros estudos, como em Henneberger et al., (2016), que demonstraram associação negativa entre a coesão familiar e o comportamento agressivo em jovens hispânicos, e a associação negativa entre o monitoramento parental e a agressividade em jovens afro-americanos. Verificar a relação entre as variáveis do estado emocional e as que mediram as relações familiares foi um dos objetivos deste estudo. Os resultados encontrados por meio da regressão múltipla demonstram a relevância das relações familiares para o estado emocional de adolescentes. As variáveis associadas foram a estrutura familiar e o conflito, assim como a afetividade e conflitos com as mães tiveram maior poder explicativo, sendo indicadores importantes para a proteção e o bem-estar dos adolescentes. Os problemas familiares são identificados como focos de desajustes para crianças e adolescentes (Henneberger et al. 2016; Hess \& Falcke, 2013). Os resultados encontrados no presente estudo identificam quais os aspectos das relações familiares têm maior peso para o estado emocional dos adolescentes. A constatação é de que o adequado funcionamento familiar se relaciona negativamente ao início de problemas de comportamento na adolescência. Os resultados sugerem que as melhorias no funcionamento da família podem ajudar a suprimir a continuidade ou o aumento de problemas comportamentais no início da adolescência (Crandall et al., 2016).

É importante considerar que a família é um sistema ativo e suas mudanças funcionam como mecanismos de desenvolvimento para os membros que a constituem. As características das relações entre os componentes da família devem ser consideradas como foco para promoção da saúde e do bem-estar de adolescentes, evitando possíveis problemas emocionais e comportamentais. Para isso, os profissionais de saúde devem observar a importância dos vínculos afetivos, relações de poder e os conflitos existentes, verificando os papéis, o nível de flexibilidade e suporte dentro do sistema familiar, assim como a comunicação. O enfoque das intervenções também deve ser ampliado para programas psicoeducativos como formas de promoção da saúde ou como manejo de desajustes, sendo que, para o último caso, o mais indicado é a modalidade de psicoterapia. Os resultados deste estudo apresentam contribuições para compreender o perfil emocional e comportamental dos adolescentes e o quanto isso está relacionado com a estrutura e o funcionamento familiar.

Apesar das contribuições apresentadas, o estudo teve as seguintes limitações: amostra de conveniência que pode ter tido como viés adolescentes mais vulneráveis, que manifestaram interesse de participar da pesquisa por desejarem estar fora da sala de aula ou mesmo por interesse de falar sobre si mesmos com alguém; falta validação dos instrumentos, uma vez que apenas dois têm estudos de validação concluídos, assim como não são apresentadas normas específicas para os adolescentes. Para conclusões mais robustas, sugerimos que as pesquisas futuras busquem minimizar as limitações e considerem a inclusão de outras 
variáveis, como personalidade do adolescente e as perspectivas de outros membros da família sobre os aspectos investigados.

\section{Referências}

Achenbach, T. M., \& Rescorla, L. A. (2001). Manual for the ASEBA school-age forms \& profiles: An integrated system of multi-informant assessment. Research Center for Children, Youth \& Families. Burlington, VT: University of Vermont.

Aggarwal, S., \& Berk, M. (2015). Evolution of adolescent mental health in a rapidly changing socioeconomic environment: A review of mental health studies in adolescents in India over last 10 years. Asian journal of psychiatry, 13, 3-12. doi:http://dx.doi.org/10.1016/j. ajp.2014.11.007

Avanci, J. Q., Assis, S. G., Oliveira, R. V., Ferreira, R. M., \& Pesce, R. P. (2007). Fatores associados aos problemas de saúde mental em adolescentes. Psicologia: Teoria e Pesquisa, 23(3), 287-294. Recuperado de http://www.scielo.br/pdf/ptp/v23n3/a07v23n3

Baptista, M. N., Baptista, A. S. D., \& Dias, R. R. (2001). Estrutura e suporte familiar como fatores de risco na depressão de adolescentes. Psicologia: Ciência e Profissão, 21(2), 5261. doi:http://dx.doi.org/10.1590/S1414-98932001000200007

Baumrind, D. (1978). Parental disciplinary patterns and social competence in children. Youth \& Society, 9(3), 239-267. Recuperado de http://journals.sagepub.com/ doi/abs/10.1177/0044118X7800900302

Cardoso, L. R. D., \& Malbergier, A. (2014). A influência dos amigos no consumo de drogas entre adolescentes. Estudos de Psicologia, 31(1), 65-73. doi:http://dx.doi. org/10.1590/0103-166X2014000100007

Cardoso, P., Rodrigues, C., \& Vilar, A. (2004). Prevalência de sintomas depressivos em adolescentes portugueses. Análise psicológica, 22(4), 667-675. Recuperado de http:// www.scielo.mec.pt/scielo.php?script=sci_arttext\&pid=S0870-82312004000400002

Conceição, L. S. L. D., \& Marques, M. O. (2012). Estilos educativos parentais (EMBU-A), sintomatologia depressiva/ansiosa, stress e autoestima, numa amostra de adolescentes (Dissertação de Mestrado, Instituto Superior Miguel Torga. Coimbra, Portugal). Recuperado de http://repositorio.ismt.pt/handle/123456789/276

Crandall, A., Ghazarian, S. R., Day, R. D., \& Riley, A. W. (2016). Maternal emotion regulation and adolescent behaviors: The mediating role of family functioning and parenting. Journal of Youth and Adolescence, 45(11), 2321-2335. doi:http://dx.doi.org/10.1007/ s10964-015-0400-3

Cruvinel, M., \& Boruchovitch, E. (2009). Autoconceito e crenças de autoeficácia de crianças com e sem sintomatologia depressiva. Interamerican Journal of Psychology, 43(3), 586593. Recuperado de http://www.redalyc.org/pdf/284/28412903019.pdf

Cunha, J. A. (2001). Manual da versão em português das Escalas Beck. São Paulo: Casa do psicólogo.

De Matos, M. B. D., Cruz, A. C. N., Dumith, S. D. C., Dias, N. D. C., Carret, R. B. P., \& Quevedo, L. D. A. (2015). Eventos estressores na família e indicativos de problemas de saúde mental em crianças com idade escolar. Ciência \& Saúde Coletiva, 20(7), 2157-2163. doi:http:// dx.doi.org/10.1590/1413-81232015207.17452014 
Dessen, M. A., \& Polonia, da C. A. (2007). A família e a escola como contextos de desenvolvimento humano. Paidéia, 17(36), 21-32. doi:http://dx.doi.org/10.1590/S0103-863X2007000100003

Ferreira, V. R. T. (2011). Perfil dos artigos sobre depressão em periódicos brasileiros. Revista de Psicologia da IMED, 3(1), 476-486. doi:http://dx.doi.org/10.18256/2175-5027

Galambos, N., Leadbeater, B., \& Barker, E. (2004). Gender differences in and risk factors for depression in adolescence: A 4-year longitudinal study. International Journal of Behavioral Development, 28(1), 16-25. doi:http://dx.doi.org/10.1080/01650250344000235

Henneberger, A. K., Varga, S. M., Moudy, A., \& Tolan, P. H. (2016). Family functioning and high risk adolescents' aggressive behavior: Examining effects by ethnicity. Journal of youth and adolescence, 45(1), 145-155. doi:http://dx.doi.org/10.1007/s10964-014-0222-8

Hess, A. R. B., \& Falcke, D. (2013). Sintomas internalizantes na adolescência e as relações familiares: Uma revisão sistemática da literatura. Psico-USF, 18(2), 263-276. doi:http:// dx.doi.org/10.1590/S1413-82712013000200010

Hoffmann, J. P., \& Cerbone, F. G. (2002). Parental substance use disorder and the risk of adolescent drug abuse: An event history analysis. Drug and Alcohol Dependence, 66(3), 255-264. doi:http://dx.doi.org/10.1016/S0376-8716(02)00005-4

Justo, L. P., \& Calil, H. M. (2006). Depressão: O mesmo acometimento para homens e mulheres? Archives of Clinical Psychiatry, 33(2), 74-79. doi:http://dx.doi.org/10.1590/ S0101-60832006000200007

Loureiro, L., Sequeira, C., Rosa, A., \& Gomes, S. (2014). Rótulos psiquiátricos "bem-mequer, mal-me-quer, muito, pouco e nada...". Revista Portuguesa de Enfermagem de Saúde Mental (Ed. Especial 1), 40-46. Recuperado de http://www.scielo.mec.pt/pdf/rpesm/ nspe1/nspe1a07.pdf

Malbergier, A., Cardoso, L. R. D., \& Amaral, R. A. D. (2012). Uso de substâncias na adolescência e problemas familiares. Cadernos de Saúde Pública, 28(4) 678-688. doi:http://dx.doi. org/10.1590/S0102-311X2012000400007

Martin, J. C. S., Hernandez-Martinez, C., Acosta, K. R., \& Domenech-Llaberia, E. (2011). Somatic complaints and symptoms of anxiety and depression in a schoolbased sample of preadolescents and early adolescents. Functional impairment and implications for treatment. Journal of Cognitive and Behavioral Psychotherapies, 11(2), 191-208. Recuperado de http://web.b.ebscohost.com/ehost/pdfviewer/ pdfviewer?vid=0\&sid=70b98ee1-5816-4bea-a8ac-f354de6dc277\%40pdc-v-sessmgr04

McGoldrick, M., Garcia-Preto, N. A., \& Carter, B. A. (2016). Expanded family life cycle: Individual, family, and social perspectives (5a ed.). Boston: Pearson.

Melo, O., \& Mota, C. P. (2013). Vinculação amorosa e bem-estar em jovens de diferentes configurações familiares. Psicologia em Estudo, 18(4), 587-597. doi:http://dx.doi. org/10.1590/S1413-73722013000400002

Mingoti, S. A. (2005). Análise de dados através de métodos de estatística multivariada: Uma abordagem aplicada. Belo Horizonte, Brasil: Editora UFMG.

Pasquali, L., Gouveia, V. V., Andriola, W. B., Miranda, F. J., \& Ramos, A. L. M. (1996). Questionário de Saúde Geral de Goldberg - Manual Técnico. São Paulo, Brasil: Casa do Psicólogo.

Pugh, K.L., \& Farrell, A.D. (2012). The Impact of Maternal Depressive Symptoms on Adolescents' Aggression: Role of Parenting and Family Mediators. J Child Fam Stud, 21, 589-602. doi:https://doi.org/10.1007/s10826-011-9511-y 
Reppold, C., \& Hutz, C. S. (2003). Prevalência de indicadores de depressão entre adolescentes no Rio Grande do Sul. Avaliaçao Psicologica: Interamerican Journal of Psychological Assessment, 2(2), 175-184. Recuperado de https://dialnet.unirioja.es/servlet/articulo?codigo=5115858 Rocha, M. M., Araújo, L. G. S., \& Silvares, E. F. M. (2008). Um estudo comparativo entre duas traduções brasileiras do Inventário de Auto-Avaliação para Jovens (YSR). Revista Psicologia-Teoria e Prática, 10(1), 14-24. Recuperado de http://pepsic.bvsalud.org/scielo. php?script=sci_arttext $\&$ pid=S1516-36872008000100002

Rosa, A., Oureiro, L. M. J., \& Sequeira, C. (2018). Literacia em saúde mental em adolescentes: Desenvolvimento de um instrumento de avaliação (Tese de Doutorado, Instituto de Ciências Biomédicas de Abel Salazar, Universidade do Porto, Porto, Portugal). Recuperado de http://oasisbr.ibict.br/vufind/Record/RCAP_52da998b7e4ba5c2a9d67900f464ebe7

Rozemberg, L., Avanci, J., Schenker, M., \& Pires, T. (2014). Resiliência, gênero e família na adolescência. Ciência \& Saúde Coletiva, 19, 673-684. doi:http://dx.doi. org/10.1590/1413-81232014193.21902013

Schenker, M., \& Minayo, M. C. D. S. (2003). A implicação da família no uso abusivo de drogas: Uma revisão crítica. Ciência \& Saúde Coletiva, 8, 299-306. doi:http://dx.doi.org/10.1590/ S1413-81232003000100022

Silva, E., \& Freire, T. (2014). Regulação emocional em adolescentes e seus pais: Da psicopatologia ao funcionamento ótimo. Análise Psicológica, 32(2), 187-198. Recuperado de http://www.scielo.mec.pt/pdf/aps/v32n2/v32n2a05.pdf

Teodoro, M. L. M. (2006). Afetividade e conflito em díades familiares: Avaliação com o familiograma. Revista Interamericana de Psicologia, 40(3), 385-390. Recuperado de http://www.redalyc.org/pdf/284/28440313.pdf

Teodoro, M. L., Allgayer, M., \& Land, B. (2009). Desenvolvimento e validade fatorial do Inventário do Clima Familiar (ICF) para adolescentes. Revista Psicologia-Teoria e Prática 11(3), 27-39. Recuperado de http://pepsic.bvsalud.org/pdf/ptp/v11n3/v11n3a04.pdf

Teodoro, M. L., Hess, A. R. B., Saraiva, L. A., \& Cardoso, B. M. (2014). Problemas emocionais e de comportamento e clima familiar em adolescentes e seus pais. Revista Psico, 45(2), 168-175. doi:10.15448/1980-8623.2014.2.13172

World Health Organization. (2018). Adolescents Mental Health. Genebra: WHO Recuperado de https://www.who.int/news-room/fact-sheets/detail/adolescent-mental-health

Recebido em: 29/08/2018

Última revisão: 09/09/2019

Aceite final: 24/09/2019

\section{Sobre os autores:}

Patrícia Martins de Freitas: Doutora em Saúde da Criança e do Adolescente pela Universidade Federal de Minas Gerais (UFMG). Professora Associada da Universidade Federal da Bahia, Instituto Multidisciplinar em Saúde. Programa de Mestrado Profissional em Psicologia da Saúde (UFBA/IMS). E-mail: pmfrei@gmail.com, Orcid: http://orcid.org/0000-0002-2065-1236

Raphael Silva Nogueira Costa: Mestre em Saúde Coletiva pela Universidade Estadual de Feira de Santana (UEFS). Docente do Centro Universitário Dom Pedro II. E-mail: raphaelsnc@gmail.com, Orcid: https://orcid.org/0000-0002-9657-6607 
Marianna Santos Rodrigues: Mestranda no Programa de Mestrado Profissional em Psicologia da Saúde na Universidade Federal da Bahia (UFBA). Especialista em Saúde da Criança e do Adolescente. Psicóloga.E-mail:mariannarodrigues.psi@gmail.com, Orcid:https://orcid.org/0000-0002-3695-2930

Bruna Rafaela de Assis Ortiz: Especialista em Terapia Intensiva. Psicóloga pela Universidade Federal do Recôncavo da Bahia (UFRB). Psicóloga Clínica. E-mail: assisbruna18@gmail.com, Orcid: https:// orcid.org/0000-0003-4958-0547

Júlio César dos Santos: Doutor em Processos de Desenvolvimento Humano e Saúde pela Universidade de Brasília (UnB). Professor Adjunto da Universidade Federal do Recôncavo da Bahia, Centro de Ciências da Saúde (UFRB/CCS). E-mail: economiapsicologia@gmail.com, Orcid: https:// orcid.org/0000-0002-9526-1136 
